\title{
Determinantes de la Adopción de Innovación en Ecuador
}

\author{
Pilamunga-Shaca, Danny ${ }^{1,2}$ iD ; Guevara-Rosero, Grace ; $^{3}$ i \\ ${ }^{1}$ Ingeniería en Ciencias Económicas y Financieras, Escuela Politécnica Nacional, Quito, Ecuador \\ ${ }^{2}$ Cooperativa de Ahorro y Crédito Mushuc Runa, Ecuador \\ ${ }^{3}$ Departamento de Economía Cuantitativa, Escuela Politécnica Nacional, Quito, Ecuador
}

\begin{abstract}
Resumen: Utilizando un modelo de estimación de elección binaria de 2 etapas, se determinan los factores que motivan a las empresas a adoptar innovaciones previamente desarrolladas en productos y procesos. Se utilizó una muestra de 8025 empresas ecuatorianas provenientes de la Encuesta de Actividades de Ciencia, Tecnología e Innovación, en sus ediciones 2009-2011 y 2012-2014. Los resultados concluyen que el recurso cognitivo de las empresas, las actitudes colaborativas con clientes y proveedores y los canales de comunicación que estas tienen influyen positivamente en la adopción de innovación. El tamaño de la empresa resultó tener una relación en forma de U, mientras que el nivel de exportaciones resultó no tener ningún efecto.
\end{abstract}

Palabras clave: Adopción, Innovación, Difusión, Conocimiento, Productos, Procesos.

\section{Determinants of the Adoption of Innovation in Ecuador}

\begin{abstract}
Using a 2-stage binary choice estimation, the factors that motivate firms to adopt previously developed innovations in products and processes are determined. A database of 8025 Ecuadorian firms from the National Survey of Science, Technology and Innovation Activities, in its 2009-2011 and 2012-2014 editions, is used. The results show that the firm's cognitive resource, collaborative attitudes with customers and suppliers and the communication channels have a positive impact on the adoption of innovation. The size of the firm has a U-shaped relation with the adoption of innovation while the level of exports does not have any effect.
\end{abstract}

Keywords: Adoption, Innovation, Diffusion, Knowledge, Products, Processes.

\section{INTRODUCCIÓN}

La innovación es uno de los componentes más importantes para el desarrollo económico, productividad y avance tecnológico de cualquier nación (Nadiri, 1993). Mediante la innovación realizada por las empresas se obtiene mayor crecimiento económico y se pueden generar mayores ventajas competitivas a largo plazo (Fagerberg, 1988). Un retraso o un estancamiento en la innovación comprometen seriamente el desempeño y la supervivencia de una economía. (Porter, 1990). La innovación en una empresa se puede lograr a través de dos vías: La primera es una innovación propia, desarrollada internamente por la misma entidad que la utiliza. La segunda opción es la adopción, que es realizada principalmente por otras entidades y adquirida posteriormente por otra empresa que hará uso de la misma (Autant-Bernard et al., 2010).

El comportamiento de adopción suele ser muy usual en países en vías de desarrollo, donde las interacciones entre clientes y empresas se ven mermadas por el bajo grado institucional, lo que hace que no existan incentivos suficientes para innovar en base al esfuerzo propio (Chaminade \& Vang, 2008). Con un análisis de costos y beneficios, algunas empresas pueden encontrar más factible adoptar una idea materializada por otros en lugar de desarrollar su propia innovación (Benhabib \& Spiegel, 2005; Nelson, 1959).

Cuando se utilizan muy frecuentemente tecnologías e ideas desarrolladas por entidades externas podría estancar el potencial innovador de las firmas nacionales, incrementando la competitividad externa y extranjera (Benhabib \& Spiegel, 2005; Liu et al., 2013; Nelson, 1959). Esto hace que las economías en vías de desarrollo se vuelvan progresivamente más dependientes de las economías más desarrolladas (Hobday, 2005; Jameson, 2016). Según el reporte The Global Innovation Index (2019) realizado por la Organización Mundial de la Propiedad Intelectual, la Universidad de Cornell y la Escuela de Negocios INSEAD, Ecuador ha ocupado consecutivamente los últimos lugares entre los países menos innovadores a nivel mundial y regional, llegando incluso a posicionarse como el sexto país menos innovador del mundo en el año 2010. De acuerdo con la Encuesta de Actividades de

*carolina.guevara@epn.edu.ec

Recibido: 01/11/2019

Aceptado: 22/09/2020

Publicado: 30/11/2020

10.33333/rp.vol46n 2.05

CC BY 4.0 
Ciencia, Tecnología e Innovación (2015), entre el año 2009 a 2014, de las 8025 empresas ecuatorianas encuestadas, 4066 introdujeron una innovación o mejora significativa de bienes, servicios o procesos, que representan el $42,07 \%$. De las empresas que innovaron, 1339 empresas (32\%) lo hicieron a través de la adopción.

El objetivo de esta investigación es analizar los determinantes de la adopción de la innovación. Para ello, se emplea la metodología Heckman-Probit que permite corregir el sesgo de selección. Este problema de sesgo consiste en que solo aquellas empresas que obtienen una innovación pueden elegir entre adopción o desarrollo propio. Esto implica que aquellas empresas que no hayan tenido alguna innovación no serían consideradas. Los datos usados pertenecen a la Encuesta de Actividades de Innovación, Ciencia y Tecnología en sus dos únicas ediciones existentes, 2009-2011 y 2012-2014.

Los resultados muestran que los canales de comunicación e intercambio creados por las empresas a través de la innovación organizacional, grupos empresariales y actividades de cooperación promueven el proceso de difusión de innovaciones, por lo cual las empresas ecuatorianas son capaces de reconocer innovaciones para posteriormente adoptarlas. El talento humano bien preparado también influye positivamente en el proceso de adopción de innovaciones, debido al efecto del intercambio y absorción de conocimiento. La participación en mercados internacionales a través de exportaciones aparenta no ser relevante.

Esta investigación contribuye a la literatura poco explorada sobre la innovación y adopción de innovaciones en vía de desarrollo, como es el caso de Ecuador, identificando la medida en la que ciertos factores inciden sobre la adopción de innovación. Además, en base a los resultados de este estudio, se proponen recomendaciones dirigidas a las entidades que tienen influencia en los procesos de innovación tanto en el ámbito público como privado.

La estructura de este estudio contempla varias secciones. En la sección 2 se realiza una exploración teórica que identifica los factores que determinan la innovación y la adopción de innovación. En la sección 3, se describen los datos y la metodología empleada. La sección 4 discute los resultados obtenidos y finalmente la sección 5 concluye y propone recomendaciones.

\section{LITERATURA RELACIONADA}

El proceso de adopción de innovación se puede entender a través de la teoría de la difusión de innovaciones (E. Rogers, 1962) y la teoría de la absorción de conocimiento (M. Cohen \& Levinthal, 1990). Rogers (1962) afirma que las innovaciones podrían generar cierta incertidumbre antes de ser lanzadas al mercado. Así, solo una minoría de empresas deciden asimilar dicho riesgo y las empresas que no tienen la capacidad de asimilar la incertidumbre pasan a ser adoptantes. El beneficio que reciban de dicha innovación decrecerá con el tiempo hasta que la innovación naturalmente pierda su valor. La segunda teoría de absorción de conocimiento muestra que la propensión a adoptar innovaciones depende de la capacidad de las firmas de asimilar y absorber conocimientos adquiridos a través del aprendizaje. A su vez, el nivel de absorción de conocimiento dependerá en gran medida del personal altamente cualificado, la estructura organizacional, estructuras de comunicación y los flujos internos de información.

Como la innovación es un factor clave para el desarrollo de los países, el estudio para países en vías de desarrollo es pertinente y relevante. Según Hobday (2005), los países en vías de desarrollo adquieren innovaciones en un tiempo tardío, influenciados principalmente por el status o liderazgo de aquellos países con mayor industrialización Kim (1997) enuncia que los países en desarrollo inicialmente adoptan tecnología externa, la asimilan después de un tiempo para posteriormente mejorarla. Este proceso es conocido como proceso de recuperación.

La adopción de innovación tiene fundamentos teóricos relacionados con el conocimiento, el aprendizaje de la empresa, los canales de intercambio y comunicación, actividades cooperativas y otras características internas. Los mecanismos de relación con cada tipo de variable se presentan a continuación.

\subsection{Nivel de Personal Altamente Cualificado}

Las empresas con mayor capital humano para innovar tendrían una mayor disposición a realizar una innovación propia respecto a tomar innovaciones desarrolladas por otros (Arundel, 2007). Sin embargo, Arrow (1962), Benhabib y Spiegel (2005), Mansfield, Schartz y Wagner (1981) y Nelson (1959) manifiestan que imitar ideas pre-existentes tiende a ser menos costoso para las empresas, a pesar de tener un elevado recurso intelectual a su disposición. Cohen y Levinthal (1990), a través de su conceptualización de capacidad de absorción de conocimiento, sugieren que aquellas entidades que cuentan con talento humano mejor preparado tienen una mayor capacidad de adquirir conocimientos externos que se tienden a explotar en la imitación de nuevos productos o procesos. Dicha capacidad de absorción depende en gran medida del nivel de conocimientos previos que posee la empresa (Jovanovic \& Rob, 1989) Nelson y Phelps (1965) argumentan que aquellos individuos con un nivel educativo más alto tienden a adoptar en mayor grado las innovaciones más productivas que aquellos con menor nivel de educación. Así, la capacidad para utilizar los resultados de los esfuerzos de investigación realizados por otras empresas o establecimientos depende de la capacidad para comprenderlos y evaluar su potencial económico. AutantBernard et al. (2010) deducen que las empresas con personal altamente cualificado deberían ser capaces de crear sus propias innovaciones y encuentran una relación negativa con la adopción de innovaciones.

\subsection{Innovación Organizacional}

Autores como Drucker (1985), Schumpeter (1934) y Hagerdorn (2002) sugieren que la innovación es resultado de la condensación de varios logros organizacionales que buscan responder a las necesidades del mercado y a la adaptación al progreso tecnológico. Así, las mejoras desarrolladas por la empresa en su sistema organizacional traen como 
consecuencia un aumento significativo de la innovación en productos y procesos (Naranjo-Valencia et al., 2012). Esto sucede puesto que las innovaciones en la organización de la empresa funcionan como mejoras hacia los canales de comunicación por las que la innovación se transmite dentro de un sistema social ${ }^{2}$ (Autant-Bernard et al., 2012; Lane \& Lubatkin, 1998; Lenox \& King, 2004). Para Veugelers (1996) y Naranjo-Valencia et al. (2012), la influencia que tenga la estructura organizacional en la decisión de desarrollar innovaciones propias o solo adoptarlas dependerá de la capacidad de absorción de conocimiento de la firma, como consideraban M. Cohen y Levinthal (1990).

\subsection{Financiamiento Público}

El soporte a través de entidades de fondos públicos parece ser efectiva para mejorar el desempeño innovador de las empresas, pues otorga apoyo a través de subvenciones, subsidios, préstamos de la banca pública con tasas de interés preferencial, exoneración de impuestos y aranceles bajos (Huergo \& Moreno, 2015). Según Autant-Bernard et al. (2010), la financiación pública de la innovación es más eficiente para fomentar la adopción que para generar la innovación en sí. Esto podría deberse a que el tipo de instrumentos financieros utilizados (subvenciones, subsidios, préstamos) hacen que, a largo plazo, las firmas generen comportamientos dependientes de otras innovaciones a pesar de tener el incentivo suficiente para desarrollar ideas por sí mismas. Esto podría también depender de la magnitud del incentivo otorgado por la banca pública, según explican Autant-Bernard et al. (2010).

Spielkamp y Rammer (2009) señalan que algunos proyectos de carácter innovador pueden resultar desfavorables para la empresa al final si se comparan las ganancias que se obtendrán contra sus costos de implementación, lo cual según B.H. Hall y Lerner (2010) representa un monto demasiado elevado para que se realice con recursos propios.

\subsection{Características Sectoriales}

La actividad principal de la empresa en el mercado determina gran parte de su comportamiento innovador (Kenessey, 1987; Landau \& Rosenberg, 1986). Los procesos de innovación requieren de fuentes de conocimiento que se desarrolla interna o externamente (M. Cohen \& Levinthal, 1990). Autores como Freeman (1988), Lamberton (1986) y Hollander (2007) concluyen que las iniciativas de desarrollo de innovaciones provienen de personal con conocimientos científicos, de diseño y de ingeniería. El aprendizaje acumulado de ciertos sectores permite mejorar las condiciones para desarrollar una innovación (M. Cohen \& Levinthal, 1990). Aquellas firmas de sectores de Alta Intensidad de Conocimiento ${ }^{3}$ poseen el talento necesario para innovar por su cuenta (Lane \& Lubatkin, 1998). Esto se debería a que las actividades de dichas empresas se basan de por sí en el desarrollo de nuevas tecnologías y de

2 El sistema social se entiende como un conjunto de individuos interrelacionados, una innovación puede ser difundida en ella si es aceptada por las firmas líderes con mayor relevancia en el mercado.

${ }^{3}$ El sector de Alta Intensidad en Conocimiento está agrupado de acuerdo a la Clasificación Industrial Internacional Uniforme (CIIU) a 2 dígitos comprende las industrias con los siguientes códigos: Q86, Q87, Q88, M69, nuevas ideas (Metcalfe \& Miles, 2000) acorde a las necesidades de sus clientes y proveedores (Savic et al., 2014).

Apriorísticamente, aquellas firmas con un alto nivel de conocimiento tendrían la capacidad de desarrollar internamente sus propias ideas (Raspe, 2009). No obstante, estas firmas también disponen de una capacidad de absorción de conocimiento externo, que permite que el aprendizaje se asimile con relativa facilidad (M. Cohen \& Levinthal, 1990). Por tanto, acoplar una innovación externa y explotarla en el mercado también puede ser una opción viable (E. Rogers, 1962).

La literatura sugiere que la tecnología es un aspecto sobresaliente de las actividades de la empresa cuando se trata de innovación y crecimiento económico (Acs \& Armington, 2004; Fagerberg, 1988; Hausmann \& Rodrik, 2003). Para Jaffe (1986), las firmas que destinan sus recursos al desarrollo tecnológico tienen mayor facilidad para lograr una innovación. Balcerowicz, P. Keczkowski y W. Katek-Rubiak (2009) sostienen que los sectores con un alto componente tecnológico son el motor de crecimiento económico del país, ya que las actividades de $\mathrm{I}+\mathrm{D}$ que se generan en estos sectores son las principales fuentes de innovación tecnológica. En síntesis, aquellas industrias de Alta Intensidad Tecnológica ${ }^{4}$ tendrían una alta probabilidad de lograr una innovación.

\subsection{Exportaciones}

Las empresas más productivas se abren caminos a mercados internacionales para mejorar los retornos de sus inversiones, aumentar su productividad y disminuir sus costos de producción (Lileeva \& Trefler, 2010). Las empresas que más destacan en su productividad e innovación serían las primeras en abrirse a mercados internacionales y comenzar a exportar (Yeaple et al., 2004). Las exportaciones funcionan como un mecanismo de aprendizaje e intercambio que permiten a las firmas locales acceder a tecnologías exteriores de última gama que posteriormente se aplican en el mercado local, lo que fomenta las actividades innovadoras locales (Albis Salas, 2015). Rogers (1962) explica a través de su teoría de la difusión de innovaciones que durante el comercio exterior existen un intercambio de activos tangibles e intangibles. Según Coe, Helpman y Hoffmaister (2009), un efecto secundario de dicho intercambio es la imitación de innovaciones. La continua interacción de las empresas exportadoras con otros países les permite acceder a nuevas tecnologías con gran eficiencia (Nobel \& Birkinshaw, 1998). Así, existe una relación positiva entre el comercio exterior y la difusión de innovaciones (Fagerberg \& Verspagen, 2002; Keller, 1997).

Respecto al intercambio de activos intangibles, a saber, ideas y conocimientos, Lucas (1989) y Autant-Bernard et al. (2010) explican que las interacciones interpersonales producidas durante el comercio exterior se relacionan directamente con la

M70, M71, M72, M73, M74, M75, K64, K65, K66, C33, E36, E39, H50, H51, J58, J59, J60, J61, J62, J63.

${ }^{4}$ El sector de Alta Intensidad Tecnológica está agrupado de acuerdo a la Clasificación Industrial Internacional Uniforme (CIIU) a 2 dígitos y abarca las industrias con los siguientes códigos: C20, C21, C26, C27, C28, C29, C30, C32. 
capacidad de absorción de conocimiento y la difusión de innovaciones. Albis y Salas (2015) y Iammarino et al. (2007) explican que la interacción y canales de comunicación creados a través del comercio pueden hacer que las empresas se limiten a aprovechar las innovaciones logradas por otras empresas más grandes. Esto es explicado por Narula y Zanfei (2003) como un efecto natural de la globalización.

Autant-Bernard et al. (2010) encuentran que el nivel de comercio exterior tiene una relación negativa con la adopción de innovaciones. Explican que esta relación era inesperada desde un principio, aunque argumentan que esto podría indicar que el efecto de incrementar la competitividad debido a la presión del comercio internacional es superior al efecto de incrementar la difusión de innovaciones. El incremento de la competitividad debido a la interacción en el comercio internacional se debe a que las firmas nacionales tienen mayor presión por incrementar su productividad, minimizando sus costos (Fagerberg \& Verspagen, 2002; Kaldor et al., 1986; Kellman, 1983).

\subsection{Tamaño de la Empresa}

Las actividades de innovación conllevan un alto costo de operación y mantenimiento que solo puede ser soportado por empresas grandes con altos volúmenes de negocio (Klepper \& M Cohen, 1996). Cohen (2010) señala que las empresas de gran tamaño generan economías a escala mediante de las actividades de $\mathrm{I}+\mathrm{D}$, a través de la adquisición de equipos especializados y contratación de personal multidisciplinario altamente capacitado, lo que conlleva a la generación de proyectos de innovación. Maskus (2000) y Battisti, Hollenstein, Stoneman y Woerter (2007) ilustran la innovación y adopción de innovación como un modelo de líder-seguidor, donde las empresas grandes son líderes y desarrollan primero una innovación y las empresas más pequeñas son seguidoras y se limitan a comprar o adquirir dicha innovación de las empresas grandes, convirtiéndose en adoptantes de innovación.

A pesar de que algunos autores concuerdan en que las firmas más grandes son las más propensas a innovar, existen otras teorías que afirman lo contrario. Tal es el caso de M. Rogers (2004), quien menciona que las empresas pequeñas tienen ciertas ventajas sobre las empresas más grandes. Las empresas más pequeñas serían más rápidas para reconocer oportunidades, más flexibles para investigar o implementar innovaciones, ajustan mejor los incentivos a sus empleados para garantizar un esfuerzo innovador óptimo, y su administración es menos rígida. En su estudio realizado para las empresas de Australia encontró que aquellas firmas pequeñas son más innovadoras en principio, no obstante, esta va decreciendo conforme aumenta el tamaño de las firmas. Otro estudio semejante fue desarrollado por B. Tether (1998), quien, utilizando los datos provenientes de la Unidad de Investigación de Políticas Científicas (Reino Unido) y Small Business Administration (EEUU), encontró que las empresas más pequeñas son las que más innovaciones tienen por empleado. Symeonidis (1996) explica que las empresas pequeñas se valen de sus ventajas competitivas en principio para incorporar innovaciones, de manera que puedan ganar una mayor participación en el mercado. No obstante, existiría un umbral en el cual la empresa se ve consolidada e innova con menos frecuencia. Autant-Bernard et al. (2010) afirman que el tamaño de la firma podría influenciar en la rentabilidad de la innovación adoptada, debido a que las empresas más grandes afrontan menores riesgos asociados a invertir en una nueva. M. Cohen y Levinthal (1990) y Dougherty y Heller (1994) insisten en que las grandes empresas adoptan con mayor frecuencia debido a su alta capacidad de absorción.

\subsection{Inversión en $I+D$}

Las actividades de Investigación y Desarrollo, también conocidas como I+D, son realizadas por las empresas con el afán de mejorar su desempeño económico. Para esto deberán especializarse e incrementar sus capacidades de aprendizaje, lo que a futuro podría derivar en la conquista de nuevos mercados (Rosenberg, 1984). La explotación de innovaciones se produce cuando la empresa hace uso eficiente de sus recursos y tiene un buen desempeño en el mercado (Schumpeter, 1934).

Autores como Buesa, Heijs y Martínez (2019) utilizan los gastos de I+D interno para explicar la probabilidad de innovación para las empresas españolas. Adicionalmente, Griliches (1979) también afirma que los gastos de I+D interno inciden directamente con la innovación.

La orientación externa en I+D de una empresa permite acceder a una variedad de mecanismos que aumentan la probabilidad de que la información sobre innovaciones ingrese al sistema organizativo de quien decide adoptar (O. Hall, 1967; Kimberly \& Evanisko, 1981). Liu et al.(2013) demuestra, a partir del caso de la economía china, que la contratación externa facilita las actividades de adquisición de innovaciones, aunque merman las capacidades locales de realizar esfuerzos internos, fortaleciendo así la competitividad extranjera. Las empresas locales que optan mayormente por realizar actividades de I+D externa se limitan a la compra o adquisición externa de innovaciones (Piga \& Vivarelli, 2004).

\subsection{Cooperación con Clientes y Proveedores}

Según Hagedoorn (2002), las empresas se ven motivadas a realizar actividades de cooperación en innovación con el fin de minimizar los costes de desarrollar una nueva idea. Para D. Arthurs y Busenitz (2006), algunas empresas con ideas innovadoras podrían tener una escasez inicial de recursos y capacidades, especialmente si estas son de reciente creación. Bruton y Rubinak (2002) enuncian que las firmas pueden superar el entorno hostil y la falta de recursos aliándose con otra empresa que, con activos complementarios, recursos humanos y tecnología única, pueden coadyuvar al desarrollo de la innovación. En un entorno cooperativo, la tarea de un proyecto de innovación podría ser repartida entre sus participantes para un trabajo conjunto (B. S. Tether \& Tajar, 2008). Así, cada firma se especializa en su área e invierte menos de lo que necesitaría si hiciera el proyecto por sí solo, generando así economías de escala (Bougrain \& Haudeville, 2002) y reduciendo significativamente los costos fijos (Gans \& Stern, 2003).

Rogers (1962) plantea que las actividades de cooperación funcionan como canales de comunicación que propagan la 
existencia de una innovación dentro del sistema social. Además, asegura que una relación eficaz con proveedores y clientes mejora las capacidades de una empresa de reconocer nuevas tecnologías, que posteriormente serán adoptadas. Para Autant-Bernard et al. (2010), la cooperación con clientes y proveedores sirve para el desarrollo de insumos complementarios que fomentan la adopción de innovaciones. Rosenberg (1972) indica que la interacción con proveedores y clientes hace que se produzca la difusión de innovaciones a través de un intercambio continuo de mejoras en las tecnologías existentes, además de un intercambio de conocimientos. Adicionalmente, Lucas (1989) está de acuerdo en que durante las interacciones con clientes y proveedores se produce un intercambio intangible de ideas, lo cual da paso a la difusión de innovaciones y vuelve a las empresas más propensas a adoptar dichas ideas.

\subsection{Grupo Empresarial}

Según Rogers (1962), la comunicación eficiente entre las empresas promueve la difusión de innovaciones puesto que se da un intercambio continuo de aprendizaje (M. Cohen \& Levinthal, 1990). Los grupos empresariales crean un mercado de financiamiento interno eficiente, con lo cual se puede acceder a fondos para desarrollar ideas con mayor facilidad, tomando prestado el dinero de otros integrantes del mismo grupo (Seru, 2007). En el mercado, la información entre empresas e inversores externos es asimétrica (Petersen \& Himmelberg, 1994), por lo que las empresas confían en el financiamiento interno del capital del grupo para sus actividades de I+D, que es menos costoso que el financiamiento del mercado externo (Myers \& Majluf, 1984). Para Belenzon y Berkovitz (2007), las empresas que se afilian a un grupo utilizan muy frecuentemente las innovaciones logradas por el resto de empresas pertenecientes al mismo grupo, por lo que, aunque la innovación no haya sido lograda por esfuerzos propios en principio, puede ser más accesible por la actitud colaborativa de las empresas dentro del grupo.

Técnicamente bastaría con que una de las empresas logre la innovación y el resto podría únicamente reutilizarla (Fisman \& Khanna, 2004) lo que sería considerada una adopción de innovación. Autant-Bernard et al. (2010) encuentran un problema puntual en la construcción de esta variable puesto que algunas empresas pueden interpretar que las innovaciones internas logradas por otras empresas del mismo grupo les pertenecen, por lo que afirmarían que ellos lograron dicha innovación por cuenta propia. En este caso, no existe alguna forma de saber si esto ocurre o no, por lo que habría que acogerse a la posibilidad del dicho error.

\subsection{Patentes}

Un factor que se debe considerar son las patentes, que son un instrumento legal que conceden el derecho exclusivo de pertenencia de una invención nueva de producto o tecnología que es susceptible de ser comercializado por un lapso determinado de tiempo (Mansfield, 1986). Este factor surte un efecto ambiguo en la innovación. Según Arrow (1962), K. Whitaker y D. Nordhaus (1970), Merges y R. Nelson (1990) y
Rai y Jagannathan (2012), el uso de un sistema de protección formal de patentes incentivaría la innovación, debido a las garantías y beneficios que el inventor recibe, como el monopolio temporal, la protección contra imitadores y los ingresos percibidos por el uso de sus patentes. En el segundo caso, según Llanes y Stefano (2010) y Boldrin y K. Levine (2012), las patentes desalientan la innovación, debido a los elevados costos de las licencias o su repercusión negativa en el equilibrio de mercado.

\section{DATOS Y METODOLOGÍA}

Para este estudio se utilizaron los datos provenientes de la Encuesta de Actividades de Ciencia, Tecnología e Innovación (ACTI) realizada por la Secretaria Nacional de Educación Superior, Ciencia y Tecnología e Innovación conjuntamente con el Instituto Nacional de Estadística y Censos (INEC), en sus ediciones 2009-2011 y 2012-2014. Unificando ambas bases de datos, se construye un pool de datos con un total de 8 025 observaciones.

La naturaleza binaria de la variable dependiente, a saber, si una empresa adopta innovación, amerita un modelo de elección binaria Probit. En este análisis, solo aquellas empresas que obtienen una innovación pudieron elegir entre adopción o desarrollo propio. Esto implica que aquellas empresas que no hayan tenido alguna innovación no serían consideradas, lo que provocaría un sesgo de selección. Para resolver el problema de sesgo de selección, se acude al método de corrección desarrollado por Heckman ${ }^{5}$ (1977). Por tanto, el método necesario para estimar la probabilidad de adoptar innovación corresponde al método Heckman-Probit. Dicho método consiste en una estimación en 2 etapas. En la primera, se estima un modelo de elección binaria Probit para la decisión de innovación, conocido como ecuación de selección, que se presenta a continuación.

$$
\operatorname{Prob}(Y=1 \mid X)=\Phi(X \beta)
$$

Donde $\mathrm{Y}$ indica el estado innovador de la empresa:

1: Si la empresa logra una innovación o mejora significativa de bienes, servicios y/o procesos.

0: caso contrario.

$\Phi$ Es la función de distribución acumulativa de la distribución normal, $X$ es un vector de variables explicativas, $\beta$ un vector de parámetros asociados a dichas variables. Las variables explicativas de la innovación, X, se describen en la Tabla 1.

Se puede denotar la ecuación (1) como un modelo de variable latente, suponiendo que existe una variable auxiliar asociado a la probabilidad de innovar, tal que:

$$
Y^{*}=X \beta+\varepsilon
$$

Se asume que el término de error asociado a la ecuación (2) está normalmente distribuido, con media cero y varianza uno, esto es:

$$
\varepsilon \sim \mathrm{N}(0,1)
$$

${ }^{5}$ Esta metodología desarrollada por James Heckam (1977) lo hizo acreedor de un premio nobel. 
A su vez, la ecuación (2) se puede representar de la siguiente forma:

$$
Y=\left\{\begin{array}{lr}
1 & \text { si } Y^{*}>0 \\
0 & \text { en otro caso }
\end{array}= \begin{cases}1 & \text { si } X \beta+\varepsilon>0 \\
0 & \text { en otro caso }\end{cases}\right.
$$

A continuación, se obtiene la probabilidad de una empresa de innovar como un modelo Probit ordinario que se estima a través de máxima verosimilitud ${ }^{6}$, representado de la siguiente manera:

Se tiene un conjunto de datos con n observaciones:

$$
\left\{x_{i} y_{i}\right\}_{i=1}^{n}
$$

Entonces, la función de máxima verosimilitud está representada por:

$$
\ln (\beta)=\sum_{i=1}^{n} y_{i} \ln \phi\left(x_{i}^{\prime} \beta\right)+\left(1-y_{i}\right) \ln \left(1-\phi\left(x_{i}^{\prime} \beta\right)\right)
$$

A partir de la ecuación (6) se obtienen los estimadores $\hat{\beta}$ :

$$
\hat{\beta}=\underset{\beta}{\arg \max } \ln L(\beta)
$$

A partir de dicha estimación se calcula el factor conocido como el Ratio Inverso de Mills, denotado por:

$$
\lambda(-\hat{x} i \hat{\beta})=\frac{\varphi\left(-\hat{x}_{i} \widehat{\beta}\right)}{\phi\left(-\widehat{x}_{l} \widehat{\beta}\right)}
$$

Donde:

$\hat{x} i$ : Valores predichos para la ecuación de innovación, cambiados a $-\hat{x}_{i} \widehat{\beta}$ por la ley de simetría de la distribución normal.

$\varphi\left(-\hat{x}_{i} \widehat{\beta}\right)$ : Función de densidad de la distribución normal evaluada en $-\hat{x}_{i} \hat{\beta}$.

$\phi\left(-\hat{x}_{i} \hat{\beta}\right)$ : Función de densidad acumulada evaluada en $-\hat{x}_{i} \hat{\beta}$

Una vez obtenido el Ratio Inverso de Mills, se añade a la especificación del modelo de adopción, dando comienzo a la segunda etapa. En esta etapa se estima el modelo de adopción de innovación, incluyendo el Ratio Inverso de Mills como una variable explicativa adicional para corregir el sesgo de selección. El modelo de la segunda etapa asociado a la probabilidad de adoptar innovación, está denotado por:

$$
\operatorname{Prob}(w=1 \mid z)=\phi(z \alpha)
$$

Donde $w$ representa si la empresa decide adoptar una innovación, lo cual no se puede observar si la empresa no logra una innovación en primer lugar, $z$ representa un vector de variables independientes y $\alpha$ un vector de parámetros, ambos evaluados en una función de distribución normal acumulada $\phi$. Las variables explicativas de la adopción de innovación se describen en la Tabla 1. Este modelo también se puede denotar como un modelo de variables latente, suponiendo que existe una variable auxiliar:

$$
W^{*}=z \alpha+\mu
$$

Al igual que con la ecuación (2), se asumen que el error asociado a la probabilidad de adoptar tiene una distribución normal, con media cero y varianza 1 .

$$
\mathrm{u} \sim \mathrm{N}(0,1)
$$

La ecuación (10) puede representarse de la siguiente manera:

$$
A=\left\{\begin{array}{lr}
1 & \text { si } W^{*}>0 \\
0 & \text { en otro caso }
\end{array}=\left\{\begin{array}{lr}
1 & z \alpha+\mu>0 \\
0 & \text { en otro caso }
\end{array}\right.\right.
$$

Como lo que se desea es obtener la probabilidad de que una empresa realice una adopción de innovación, dado que introdujo alguna innovación, y, una vez añadido el Ratio Inverso de Mills como una nueva variable explicativa adicional, se tiene:

$$
E[W=1 \mid z, Y=1]=\phi\left[z \alpha+\rho \sigma_{u} \wedge(-\hat{x} i \hat{\beta})\right]
$$

Donde:

$\phi$ : Función de distribución acumulativa de la distribución normal.

$\rho$ : Correlación entre los factores no observados de la propensión a innovar $\varepsilon$ y de la propensión a adoptar $u$.

$\sigma_{\mathrm{u}}$ : Desviación estándar de $u$

ᄉ: Ratio Inverso de Mills evaluada en $-\hat{x} i \hat{\beta}$.

Los parámetros para la ecuación de adopción se estiman a través de máxima verosimilitud, de la misma manera que la ecuación (6) y (7).

Según Heckman (1977), como $\sigma_{\mathrm{u}}>0$, el coeficiente asociado a 入 solo puede ser cero si $\rho=0$, con lo que probar la hipótesis nula de que el coeficiente de $\lambda$ es cero equivale a probar la selectividad de la muestra.

\subsection{Descripción de Variables}

Al tratarse de una estimación en 2 etapas, existen entonces 2 variables independientes, en la ecuación de innovación viene representada de la siguiente manera:

$$
Y=\left\{\begin{array}{lr}
1 & \text { si introdujo una innovación } \\
0 & \text { caso contrario }
\end{array}\right.
$$

Se desarrollarán 3 estimaciones econométricas, por lo cual habrá 3 variables independientes, una que explique la propensión a innovar en productos, otra en proceso y una que abarque ambos casos simultáneamente. La variable dependiente de la segunda etapa también contemplará aquellos 3 casos y se representa de la siguiente manera:

$$
Y= \begin{cases}1 & \text { si adopta la innovación } \\ 0 & \text { caso contrario }\end{cases}
$$

A continuación, se citan todas las variables independientes que serán utilizadas en el modelo econométrico, cada una incluirse en la ecuación de primera etapa, segunda etapa o en ambos casos simultáneamente.

\footnotetext{
${ }^{6}$ Método para estimar los parámetros no conocidos $\hat{\beta}$ desarrollado por Aldrich (1997)
} 
Tabla 1. Variables Independientes

\begin{tabular}{|c|c|}
\hline $\begin{array}{c}\text { Variable } \\
\text { Independie } \\
\text { nte }\end{array}$ & Descripción \\
\hline \multicolumn{2}{|c|}{ Afectan a la Innovación y Adopción de Innovaciones } \\
\hline $\begin{array}{l}\text { Nivel de Personal } \\
\text { Altamente Cualificado }\end{array}$ & $\begin{array}{l}\text { Número de trabajadores } \\
\text { con título de tercer nivel o } \\
\text { superior. }\end{array}$ \\
\hline Innovación Organizacional & $\begin{array}{l}\text { Igual a } 1 \text { si la empresa } \\
\text { obtuvo una innovación } \\
\text { organizacional, } 0 \text { caso } \\
\text { contrario. }\end{array}$ \\
\hline Financiamiento Público & $\begin{array}{l}\text { Igual a } 1 \text { si la firma recibió } \\
\text { financiamiento público, } 0 \\
\text { caso contrario. }\end{array}$ \\
\hline $\begin{array}{l}\text { Alta Intensidad } \\
\text { en } \\
\text { Conocimiento }\end{array}$ & $\begin{array}{l}\text { Igual a } 1 \text { si la empresa } \\
\text { pertenece a una industria } \\
\text { intensiva en conocimiento, } \\
0 \text { caso contrario. }\end{array}$ \\
\hline $\begin{array}{l}\text { Ln (Exportaciones } \\
\text { Promedio) }\end{array}$ & $\begin{array}{l}\text { Promedio de exportaciones } \\
\text { en los } 3 \text { últimos años en } \\
\text { logaritmo. }\end{array}$ \\
\hline
\end{tabular}

Afectan Únicamente a la Innovación

Ln (Ventas Promedio) Promedio de las ventas en los 3 últimos años en logaritmo.

$(\operatorname{Ln}(\text { Ventas Promedio }))^{\wedge} 2$ Promedio de las ventas en los 3 últimos años en logaritmo elevado al cuadrado.

Ln (I+D Interna Promedio) Promedio de los gastos en I+D interna de los 3 últimos años en logaritmo.

Patentes Igual a 1 si la firma ha emprendido búsqueda de patentes, 0 caso contrario. Alta Intensidad Igual a 1 si la empresa Tecnológica pertenece a una industria intensiva en tecnología, 0 caso contrario.

Afectan Únicamente a la Adopción de Innovaciones

Ln (Ventas Promedio Ponderadas)

Promedio de ventas en los 3 últimos años ponderadas de acuerdo al sector al que pertenecen en logaritmo.

Ln (Ventas Promedio Ponderadas) ${ }^{\wedge} 2$ Promedio de ventas en los 3 últimos años ponderadas de acuerdo al sector al que pertenecen en logaritmo elevado al cuadrado.

Ln (I+D Externa

Promedio)

Promedio de los gastos en I+D externa de los 3 últimos años en logaritmo.

Cooperación con Clientes y Igual a 1 si la empresa Proveedores. realizó cooperación con clientes o proveedores, 0 caso contrario.

Grupo Empresarial empresarial, 0 caso contrario

Se ha optado por ponderar el volumen de ventas respecto al sector en el que pertenecen, esto con el fin de diferenciar la ecuación de selección de la ecuación de adopción de innovaciones, en concordancia con la metodología aplicada por Autant-Bernard et al. (2010) para la economía de la Unión Europea.

\section{RESULTADOS}

En la siguiente tabla se presentan las estimaciones de los modelos Heckman-Probit. En la parte superior de la Tabla 2 se muestran los efectos marginales de la ecuación de selección de innovación de la primera etapa y en la parte inferior se muestran los resultados de la ecuación de la adopción de la segunda etapa. En la columna (1) se muestra el modelo que abarca las innovaciones en productos y procesos, en la columna (2) se muestra el modelo de innovación en productos y en la columna (3) es el modelo de innovación en procesos.

Tabla 2. Resultados de las Estimaciones Econométricas Heckman-Probit

\begin{tabular}{|c|c|c|c|}
\hline & (1) & (2) & (3) \\
\hline Variable & General & Productos & Procesos \\
\hline \multicolumn{4}{|l|}{ Adopción } \\
\hline $\begin{array}{l}\text { Ln (Ventas } \\
\text { Promedio } \\
\text { Ponderadas) }\end{array}$ & $\begin{array}{c}-0,0353 * * \\
(0,0101)\end{array}$ & $\begin{array}{c}-0,0416^{* * * *} \\
(0,0661)\end{array}$ & $\begin{array}{l}-0,0152 \\
(0,0643)\end{array}$ \\
\hline $\begin{array}{c}\text { Ln (Ventas } \\
\text { Promedio } \\
\text { Ponderadas) } \\
\wedge 2\end{array}$ & $\begin{array}{c}0,0020^{* * * *} \\
(0,0023)\end{array}$ & $\begin{array}{c}0,0022 * * * \\
(0,0022)\end{array}$ & $\begin{array}{c}0,0008 \\
(0,0101)\end{array}$ \\
\hline $\begin{array}{c}\text { Ln (I+D } \\
\text { Externa } \\
\text { Promedio) }\end{array}$ & $\begin{array}{c}0,0248 * * * \\
(0,0098)\end{array}$ & $\begin{array}{c}0,0167 * * * \\
(0,0112)\end{array}$ & $\begin{array}{c}0,0190 * * * \\
(0,0101)\end{array}$ \\
\hline $\begin{array}{l}\text { Nivel de } \\
\text { Personal } \\
\text { Altamente }\end{array}$ & $\begin{array}{c}0,0001 * * * \\
(0,0001)\end{array}$ & $\begin{array}{c}0,0001 * * \\
(0,0002)\end{array}$ & $\begin{array}{c}0,0002 * * * \\
(0,0001)\end{array}$ \\
\hline $\begin{array}{c}\text { Cualificado } \\
\text { Innovación } \\
\text { Organizacion } \\
\text { al }\end{array}$ & $\begin{array}{c}0,0756 * * * \\
(0,0506)\end{array}$ & $\begin{array}{c}0,0264 \\
(0,0649)\end{array}$ & $\begin{array}{c}0,0958 * * * \\
(0,0587)\end{array}$ \\
\hline $\begin{array}{c}\text { Financiamient } \\
\text { o Público }\end{array}$ & $\begin{array}{c}0,1062 * * * \\
(0.1236)\end{array}$ & $\begin{array}{c}0,0583 \\
(0,1485)\end{array}$ & $\begin{array}{c}0,1098 * * * \\
(0,1386)\end{array}$ \\
\hline $\begin{array}{c}\text { Alta } \\
\text { Intensidad en } \\
\text { Conocimiento }\end{array}$ & $\begin{array}{c}0,0917 * * * \\
(0,0568)\end{array}$ & $\begin{array}{c}0,1140 * * * \\
(0,0712)\end{array}$ & $\begin{array}{c}0,6080 * * * \\
(0,0643)\end{array}$ \\
\hline $\begin{array}{l}\text { Cooperación } \\
\text { con Clientes y } \\
\text { Proveedores }\end{array}$ & $\begin{array}{c}0,1006 * * * \\
(0,0574)\end{array}$ & $\begin{array}{c}0,0765 * * * \\
(0,0079)\end{array}$ & $\begin{array}{c}0,0876 * * * \\
(0,0642)\end{array}$ \\
\hline $\begin{array}{c}\text { Grupo } \\
\text { Empresarial }\end{array}$ & $\begin{array}{c}0,1084 * * * \\
(0,0609)\end{array}$ & $\begin{array}{c}0,0844 * * * \\
(0,0777)\end{array}$ & $\begin{array}{c}0,1160 * * * \\
(0,0664)\end{array}$ \\
\hline $\begin{array}{l}\text { Ln } \\
\text { Exportaciones } \\
\text { Promedio }\end{array}$ & $\begin{array}{c}-0,0055^{* * *} \\
(0,0053)\end{array}$ & $\begin{array}{l}-0,0045 \\
(0,0070)\end{array}$ & $\begin{array}{c}-0,0041 * * \\
(0,0085)\end{array}$ \\
\hline $\begin{array}{c}\text { Inversa de } \\
\text { Mills }\end{array}$ & $\begin{array}{c}0,0887 * * * \\
(0,0594)\end{array}$ & $\begin{array}{c}0,0590 * * * \\
(0,0773)\end{array}$ & $\begin{array}{c}0,1386^{* * * *} \\
(0,0811)\end{array}$ \\
\hline $\begin{array}{c}\text { Nro. de } \\
\text { empresas } \\
\text { innovadoras }\end{array}$ & 3376 & 2246 & 2585 \\
\hline Innovación & & & \\
\hline $\begin{array}{l}\text { Ln (Ventas } \\
\text { Promedio) } \\
\text { Ln (Ventas } \\
\text { Promedio) ^2 }\end{array}$ & $\begin{array}{c}0,0424 * * * \\
(0,0585) \\
-0,0019 * * * \\
(0,0021)\end{array}$ & $\begin{array}{c}0,0084 \\
(0,0559) \\
-0,0069 \\
(0,0020)\end{array}$ & $\begin{array}{c}0,0460 * * * \\
(0,0614) \\
-0,0016 \\
(0,0022)\end{array}$ \\
\hline $\begin{array}{l}\text { Ln (I+D } \\
\text { Interna } \\
\text { Promedio) }\end{array}$ & $\begin{array}{c}0,1463 * * * \\
(0,0152)\end{array}$ & $\begin{array}{c}0,0590 * * * \\
(0,0071)\end{array}$ & $\begin{array}{c}0,0631 * * * \\
(0,0072)\end{array}$ \\
\hline $\begin{array}{c}\text { Nivel de } \\
\text { Personal } \\
\text { Altamente } \\
\text { Cualificado }\end{array}$ & $\begin{array}{c}0,0005 * * * \\
(0,0002)\end{array}$ & $\begin{array}{c}0,0002 * * * \\
(0,0017)\end{array}$ & $\begin{array}{c}0,0003 * * * \\
(0,0002)\end{array}$ \\
\hline $\begin{array}{c}\text { Innovación } \\
\text { Organizacion } \\
\text { al }\end{array}$ & $\begin{array}{c}0,2245 * * * \\
(0,0356)\end{array}$ & $\begin{array}{c}0,1380 * * * \\
(0,0354)\end{array}$ & $\begin{array}{c}0,2008 * * * \\
(0,0344)\end{array}$ \\
\hline $\begin{array}{c}\text { Financiamient } \\
\text { o Público }\end{array}$ & $\begin{array}{c}0,4920 * * * \\
(0,2034)\end{array}$ & $\begin{array}{c}0,3500 * * * \\
(0,1213)\end{array}$ & $\begin{array}{c}0,3658 * * * \\
(0,1266)\end{array}$ \\
\hline $\begin{array}{c}\text { Alta } \\
\text { Intensidad en } \\
\text { Conocimiento }\end{array}$ & $\begin{array}{c}0,0897 * * * \\
(0,0395)\end{array}$ & $\begin{array}{c}0,1418 * * * \\
(0,0389)\end{array}$ & $\begin{array}{r}0,0273 * * \\
(0,0391)\end{array}$ \\
\hline
\end{tabular}




\begin{tabular}{cccc}
$\begin{array}{c}\text { Alta } \\
\text { Intensidad }\end{array}$ & $0,0750^{* * *}$ & $0,1263 * * *$ & $0,0620 * * *$ \\
Tecnológica & $(0,0764)$ & $(0,0719)$ & $(0,0709)$ \\
Patente & $0,2065^{* * *}$ & $0,1867 * * *$ & $0,1373 * * *$ \\
Ln & $(0.0613)$ & $(0,0569)$ & $(0,0558)$ \\
(Exportacione & 0.0017 & 0,0013 & 0,0012 \\
s Promedio) & $(0,0038)$ & $(0,0354)$ & $(0,0037)$ \\
$\rho$ & 0,2684 & 0,1977 & 0,4115 \\
& chi2(1)= & chi2(1) & chi2 $2(1)=$ \\
LR test ${ }^{7}(\rho$ & 18,72 & $=6,61$ & 26.58 \\
$=0):$ & Prob>chi2 & Prob>chi2 & Prob>chi2 \\
$\begin{array}{c}\text { Correcta } \\
\text { clasificación }\end{array}$ & 68,0000 & $=0,0102$ & $=0,0000$ \\
N & $8025 \%$ & $76,27 \%$ & $69,86 \%$ \\
\hline
\end{tabular}

Se añaden el error estándar y el efecto parcial, este último representa el cambio que experimenta la variable dependiente de cada ecuación por cada unidad adicional de esa variable independiente. Entre paréntesis se encuentra el error estándar correspondiente a cada variable. El nivel de significancia se visualiza de acuerdo al número de asteriscos $(* * *=0 \%$; $* *=5 \% ; *=10 \% ; \sin$ asteriscos $=$ no es significativo)

En el modelo general para productos y procesos, todas las variables resultaron ser significativas a un $95 \%$ de confianza, tanto en innovación como en adopción, a excepción de las exportaciones, los cuales no resultaron ser significativas en innovación. Como el coeficiente relacionado a la Ratio Inverso de Mills es significativo, se rechaza la hipótesis de la nulidad de la correlación entre los factores no observados de la propensión a innovar y de la propensión a adoptar. Así, se corrigió exitosamente el sesgo de selección a través de la metodología Heckman-Probit.

El tamaño de las empresas medido a través del volumen de ventas muestra tener una relación con la adopción de innovación en forma de U como se muestra en la Figura 1. El incremento por cada unidad adicional de este factor no es lineal, sino que depende de las ventas ${ }^{8}$. Las empresas pequeñas tienen menor propensión a adoptar innovaciones en principio hasta alcanzar un umbral de aproximadamente \$1 468 en ventas anuales 9 , a partir del cual aumenta la propensión a adoptar innovaciones. Como el umbral estimado es relativamente bajo, se puede decir que la mayoría de empresas tienden a adoptar innovaciones. La alta capacidad de absorción de las empresas grandes les permite adoptar innovaciones (Autant-Bernard et al., 2010; Dougherty \& Heller, 1994; M. Cohen \& Levinthal, 1990). Este factor aparenta no tener efecto cuando se trata de adopción de innovación en procesos.

\footnotetext{
${ }^{7}$ Este test tiene por hipótesis inicial la correlación entre los factores no observados de la propensión a innovar $\varepsilon$ y de la propensión a adoptar $u$ es cero, es decir, que el coeficiente correspondiente al Ratio Inverso de Mills es cero, que implicaría que no hubo sesgo de selección que debió corregirse, al rechazarse esta hipótesis implicaría que la metodología fue apropiada.

${ }^{8}$ La función está definida por: $\frac{\Delta \operatorname{Prob}\left(Y=1 \mid X_{i k}\right)}{\Delta\left(\ln \left(X_{k}\right)\right)}=\Phi\left(X_{i} B\right) *\left(B_{1}+2 * B_{2} * \ln \left(X_{k}\right)\right)$ donde: $Y$ : Variable independiente (Innovación o adopción)

$X_{k}$ : Variable independiente de la cual se busca obtener la relación marginal (ventas en logaritmos)
}

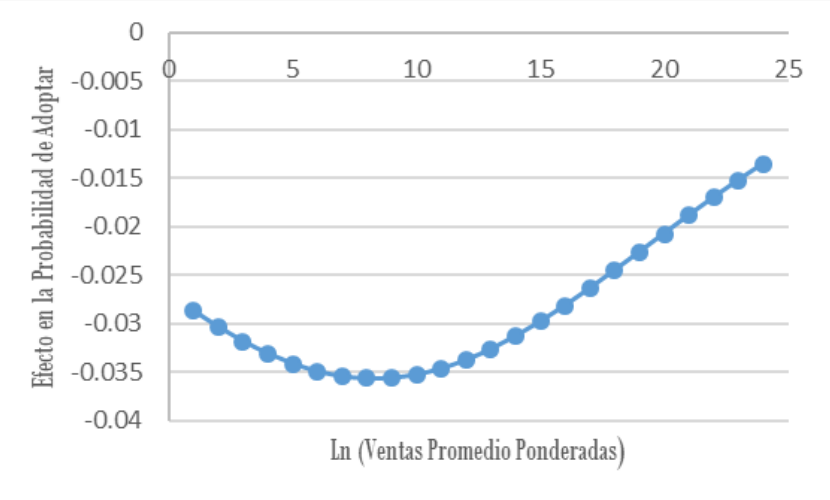

Figura 1. Efecto marginal del tamaño de la empresa sobre la adopción de innovaciones

Respecto a la innovación se obtuvo una relación en forma de u invertida con el tamaño de la empresa como se muestra en la Figura 2. Conforme la empresa va creciendo, alcanza un umbral de tamaño de aproximadamente \$61960026 en ventas anuales, posteriormente se ve consolidada dentro del mercado y su innovación es cada vez menor. Como el umbral es alto, se puede decir que la mayoría de empresas incrementan su nivel de innovación conforme crecen (M. Rogers, 2004; B. Tether, 1998). Dentro de la innovación en productos, esta variable no resultó relevante.

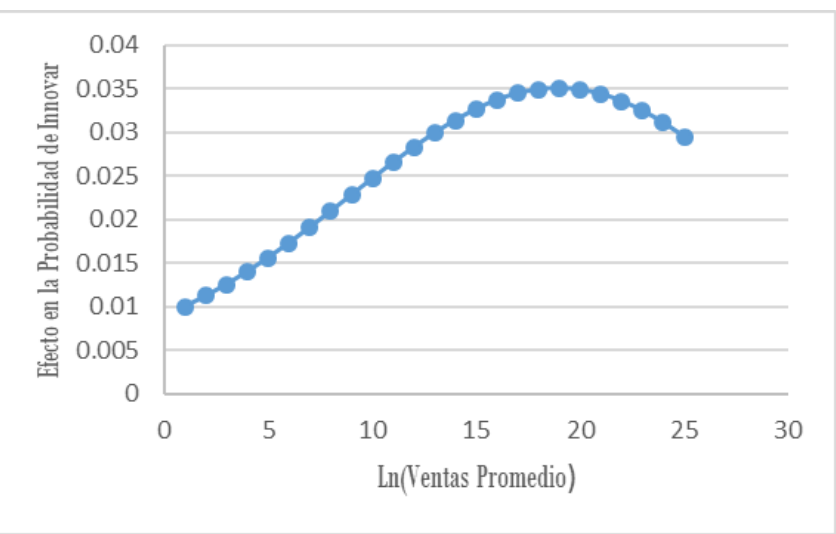

Figura 2. Efecto marginal entre el tamaño de la empresa y la innovación

El nivel de gastos en I+D externo resultó tener un signo positivo con la adopción, es decir, que mientras una empresa incremente sus recursos destinados a I+D de origen externo también incrementará su propensión a adoptar una innovación, ya sea de productos o procesos, en concordancia con Kimberly y Evanisko (1981), Liu et al. (2013) y O. Hall (1967). Un incremento en $1 \%$ en los gastos de I+D externa incrementa la propensión de la empresa a adoptar una innovación en productos en $0,017 \%$ y en procesos en $0.019 \%$. Los gastos en $\mathrm{I}+\mathrm{D}$ interna resultaron tener un efecto positivo en la innovación, en concordancia con la teoría citada por

$X_{i}$ : Variables Independientes

$B$ : Coeficiente relacionado a la variable independiente

$\Phi:$ Función de densidad de la distribución normal

${ }^{9}$ Cuando el logaritmo natural de las ventas anuales promedio llega a ser de 7.292, el efecto marginal respecto a la probabilidad de adoptar comienza a ser creciente. Aplicando la función inversa del logaritmo natural, las ventas resultan ser de $\$ 1.468$. 
Rosenberg (1984), Griliches (1979) y la evidencia empírica de Buesa et al. (2019). Un incremento en $1 \%$ en los gastos de I+D interna de la empresa incrementa la propensión de la empresa a innovar en productos en $0,059 \%$ y en procesos en $0,063 \%$.

La pertenencia al sector de Alta Intensidad en Conocimiento muestra tener una relación positiva con la adopción. Aquellas empresas que pertenecen a este sector tienen mayor propensión de 9,17\% a adoptar innovaciones en comparación a aquellas que no pertenecen a dicho sector ${ }^{10}$. Esto se debe a sus altos niveles de conocimiento técnico, científico y su alta capacidad de absorción de conocimiento, en concordancia con Rogers, (1962) y M. Cohen y Levinthal, (1990). Se puede apreciar a su vez que el efecto es mucho mayor en la propensión de adoptar innovaciones en procesos $(60,8 \%)$ que en productos $(11,40 \%)$, lo cual indica que el recurso intelectual de la empresa es mucho más efectivo en la absorción de conocimiento para la adopción de procesos que en productos (M. Cohen \& Levinthal, 1990), que va directamente relacionado en mejoras en los procesos de la organización. La incidencia sobre la probabilidad de innovar de la pertenencia a un sector con alta intensidad de conocimiento es mayor para la innovación propia en productos (14\%) que para la innovación propia en procesos $(2,7 \%)$, en concordancia con Lamberton (1986), Hollander (2007), Freeman (1988), Metcalfe y Miles (2000) y M. Cohen y Levinthal (1990). Las empresas del sector de Alta Intensidad Tecnológica son más propensas a innovar en $7,61 \%$ en comparación a aquellas que no pertenecen a este sector. El efecto es superior para la propensión a innovar en productos $(12,6 \%)$ que para la propensión en innovar en procesos $(6,2 \%)$. Esto se explica porque la disposición de tecnologías se destina principalmente a la innovación en productos, de acuerdo con Savic et al., (2014), Z. Acs y Armington (2004), Hausmann (2003), Fagerberg (1988), Jaff (1986), Balcerowicz P. Keczkowski y W. Katek-Rubiak (2009).

El nivel de personal altamente cualificado también resultó tener un efecto positivo con la adopción de innovaciones. Las empresas ecuatorianas estarían absorbiendo el conocimiento externo e interno, que es posteriormente asimilado y utilizado para incorporar eficientemente las innovaciones adoptadas dentro de sus instalaciones (M. Cohen \& Levinthal, 1990; Nelson \& Phelps, 1965). Por cada unidad adicional en el personal altamente cualificado de la empresa, la propensión a adoptar innovaciones en producto incrementa en un $0.01 \%$ y en proceso en un $0.02 \%$. En lo que corresponde a innovación, el hecho de poseer un mayor personal con un alto nivel de preparación académico también hace que las firmas tengan mayores oportunidades de innovar. El incremento que percibe la innovación por cada unidad adicional en este factor es de $0,05 \%$. El recurso intelectual de las empresas resulta beneficioso para el comportamiento innovador de las empresas en concordancia con Lundvall (2019) y Bordogna, Fromm y Ernst (1993) y E. Porter y Stern (2000).

Haber logrado una innovación organizacional tuvo una relación positiva con la adopción de innovaciones. Este resultado implicaría que las mejoras en la estructura organizacional influyen en los procesos de comunicación interna de la empresa, que, consecuentemente, incrementan su capacidad de absorción de conocimiento, lo cual surte efecto en incrementar su propensión a adoptar innovaciones, estos resultados concuerdan con las teorías de Autant-Bernard et al. (2010), Rogers (1962), Lane y Lubatkin (1998) y Lenox y King (2004). En término de efectos marginales, si una empresa innovadora incorpora una innovación organizacional la probabilidad de que adopte una innovación incrementa en un $7,56 \%$. No obstante, este aspecto no fue relevante para explicar la adopción de innovación en productos.

Respecto a la innovación también se obtuvo un efecto positivo, las empresas que hayan logrado incorporar alguna innovación organizacional en sus instalaciones tendrían una mayor capacidad de innovar en productos y/o procesos, acorde con lo mencionado por Drucker (1985), Hagedoorn (2002), Schumpeter (1934), Lugones et al. (2019), Damanpour y Gopalakrishnan (2001) y Naranjo-Valencia et al. (2012). Específicamente su probabilidad de innovar incrementa en un $22,45 \%$.

El financiamiento público resultó tener un signo positivo con la innovación, es decir que los instrumentos utilizados por el gobierno (subsidios, exoneraciones, créditos, etc.) cumplen su objetivo de promover la innovación, que también vuelve a las empresas más susceptibles de adoptar dicha innovación. El hecho de que una empresa innovadora reciba financiamiento público hace que esta incremente su propensión a adoptar esa innovación en un $10.62 \%$. Particularmente se obtuvo el mismo resultado que el estudio empírico de Autant-Bernard et al. (2010). Este aspecto no resulta relevante para explicar la adopción de innovación en productos. En cuanto a la innovación, el incremento adicional por tener financiamiento público es de 49,20\%.

El hecho de pertenecer a un grupo empresarial incrementa la propensión a adoptar una innovación en un $10,84 \%$. Esto se debe a la ventaja de poder acceder a las nuevas tecnologías de las otras empresas que pertenecen al grupo, el mercado financiero interno, la actitud colaborativa, y el intercambio de aprendizaje que promueve la difusión de innovaciones, en concordancia con Belenzon y Berkovitz, (2007), M. Cohen y Levinthal, (1990) y Autant-Bernard et al. (2010). Adicionalmente, el efecto es significativamente mayor en procesos que en productos, ya que la pertenencia a un grupo empresarial está asociado a los cambios en la estructura organizacional de la empresa, que deriva en cambios y mejoras en los procesos (Autant-Bernard et al., 2010).

Las actividades de cooperación con clientes y proveedores tuvieron en efecto positivo con la adopción de innovaciones. Aquellas empresas que realizan estas actividades de cooperación incrementarían su propensión a adoptar innovaciones en un $10,06 \%$. Este factor funciona como una fuente de conocimiento que forman canales de intercambio y comunicación entre empresas que propagan la existencia de una innovación, dando lugar al proceso de difusión de innovaciones, lo que permite a las empresas reconocer e

\footnotetext{
${ }^{10} \mathrm{Su}$ interpretación se realiza con respecto a aquellas industrias que no se encuentran en este sector
} 
incorporar innovaciones según las teorías citadas por Rogers (1962), Autant-Bernard et al. (2010), Rosenberg (1972) y Lucas (1989).

Las empresas que participan en los mercados internacionales a través de la exportación no ven incrementada su innovación, ya sea por adopción o por desarrollo propio, lo que implicaría que el intercambio de recursos tangibles e intangibles que se producen en el comercio exterior a través de canales de difusión citados por Rogers (1962), Coe et.al. (2009), Fagerberg y Verspagen (2002) y Keller (1997) y la presión sobre la competitividad de las empresas enunciado por AutantBernard et al. (2010), Kaldor, Sharp y Walker (1986) y Kellman (1983) no surten efecto.

Las patentes resultaron tener un efecto positivo en la innovación, es decir, que los beneficios como el monopolio temporal, la protección contra imitadores y el cobro por licencias son aprovechados por las firmas ecuatorianas para impulsar la innovación, dichos resultados concuerdan con Arrow (1962), K. Whitaker y D. Nordhaus (1970), Gedik (2012), Merges y R. Nelson, (1990), Rai y Jagannathan, (2012). Aquellas empresas que emprenden la búsqueda de patentes incrementan su propensión a innovar en un $20,65 \%$.

\section{CONCLUSIONES Y RECOMENDACIONES}

A través de un análisis descriptivo, se pudo verificar que Ecuador no ha tenido un posicionamiento favorable en comparación al resto del mundo en temas de innovación. Esto puede deberse al bajo nivel de innovación de las empresas ecuatorianas que mayoritariamente realizan innovaciones de mejora significativa o no innovaciones nuevas. Otro aspecto importante del comportamiento innovador de las empresas es que una gran parte de ellas adoptan innovación. Con el objetivo de identificar los factores que incentivan la adopción, se empleó un modelo Probit. Los resultados mostraron que los grupos empresariales, la innovación organizacional y la cooperación con clientes o proveedores promueven la creación de canales de intercambio de recursos tangibles e intangibles que propagan las innovaciones y facilitan la adopción de las mismas. El personal altamente cualificado puede también promover la adopción de innovaciones. En un entorno de industrias con requerimientos científicos altos, las empresas tienen una alta probabilidad de adoptar innovación. Las empresas ecuatorianas parten siendo bastante innovadoras hasta alcanzar un umbral en ventas de \$61960 026 anuales, momento desde el cual se ve consolidado en el mercado y empiezan a innovar cada vez menos. En cuanto a la adopción, las empresas comienzan a hacerlo a partir de un bajo umbral de solo \$1 468 en ventas anuales. Los recursos que las empresas ecuatorianas destinen a la Investigación y Desarrollo de origen externo harían que se vuelvan más susceptible en adoptar innovaciones. La propensión a generar innovaciones propias incrementaría con la inversión en recursos de I+D interna. La presión de la competitividad internacional y el intercambio de tecnologías y conocimientos que se producen con las exportaciones no influyen en el comportamiento innovador de las empresas ecuatorianas. El apoyo gubernamental promueve la innovación y la adopción de innovaciones como resultado de los instrumentos financieros otorgados por entidades públicas.
Las patentes tienen la capacidad de promover la innovación en lugar de desincentivarla, este efecto puede deberse a sus beneficios asociados como el monopolio temporal, protección contra imitadores y costo de las licencias de uso. Las industrias que requieren un uso intensivo de tecnología en sus instalaciones tienen una mayor propensión a innovar, tanto en productos como en proceso.

\subsection{Recomendaciones}

En función al objetivo y resultados de esta investigación, se proponen recomendaciones dirigidas a sectores influyentes en tecnología y desarrollo de innovaciones, tanto públicos como privados. Para aumentar la propensión de las empresas a innovar y adoptar innovación, se debe incentivar la formación del capital humano. Para ello, se debe impulsar el acceso de la población a la educación superior de calidad. Así, los trabajadores capacitados aumentarán la capacidad de absorción y generación de conocimiento de las empresas para adoptar innovaciones o innovar con mayor facilidad. Otro factor clave a impulsar es la inversión de investigación y desarrollo tanto interna como externa. En este aspecto, el contexto actual indica que las empresas tienen poco interés en este tipo de inversión. Solo un $22.7 \%$ invierte en I+D en los años 2011 y 2014. Para mejorar este indicador, se necesita impulsar la cooperación con las universidades. En vista de que las actividades colaborativas como las agrupaciones empresariales y la cooperación con clientes y proveedores tienen una influencia positiva en la adopción de innovación, es necesario aprovechar al máximo los canales de comunicación e intercambio por los cuales se transfiere la existencia de una innovación. De esta manera, las empresas ecuatorianas podrían incorporarlas eficientemente dentro de sus firmas y obtener beneficios. Dado que la proporción de empresas innovadoras decrece, es recomendable para los entes influyentes en los sectores productivos desarrollar programas $\mathrm{y}$ nuevos incentivos que motiven a las empresas a innovar.

Esta investigación sugiere algunas pistas de investigación futura. Una de ellas es la consideración de la intensidad de adopción de innovación para conocer en qué medida las empresas adoptan innovación y si esta es mayor que la innovación propia. Otra línea de investigación es discernir sobre la importancia relativa de los efectos de la adopción de la innovación respecto a los de la innovación propia, analizando variables de rendimiento.

\section{REFERENCIAS}

Acs, Z., \& Armington, C. (2004). Employment Growth and Entrepreneurial Activity in Cities. Regional Studies, 38, 911-927. https://doi.org/10.1080/0034340042000280938

Albis Salas, N. (2015). Determinantes de la innovación y la productividad en las subsidiarias extranjeras y las empresas exportadoras en la industria en Colombia. Revista de Estudios Empresariales. Segunda Época, 2. https://doi.org/10.17561/ree.v0i2.2740

Aldrich, J. (1997). R. A. Fisher and the Making of Maximum Likelihood 1912-22. Statistical Science, 12 (3), 162176. 
Arrow, K. (1962). Economic Welfare and the Allocation of Resources for Invention. In The Rate and Direction of Inventive Activity: Economic and Social Factors (pp. 609-626). National Bureau of Economic Research, Inc. https://econpapers.repec.org/RePEc:nbr:nberch:2144

Arundel, A. (2007). Innovation survey indicators: What impact on innovation policy? Science, Technology and Innovation Indicators in a Changing World: Responding to Policy Needs.

Autant-Bernard, C., Billand, P., \& Massard, N. (2012). Innovation and Space From Externalities to Networks. Knowledge and Talent in Regional and Global Context.

Autant-Bernard, C., Guironnet, J.-P., \& Massard, N. (2010). The Determinants of Innovation Adoption. SSRN Electronic Journal. https://doi.org/10.2139/ssrn.1731550

Balcerowicz, E., P\keczkowski, M., \& Wzilkatek-Kubiak, A. (2009). The innovation patterns of firms in low and high technology manufacturing sectors in the new member states (Issue 390). Center for Social and Economic Research (CASE). http://hdl.handle.net/10419/128191

Battisti, G., Hollenstein, H., Stoneman, P., \& Woerter, M. (2007). Inter and Intra firm diffusion of ICT in the United Kingdom (UK) and Switzerland $(\mathrm{CH})$. An internationally comparative study based on firm-level data. Economics of Innovation and New Technology, 16(8), 669-687.

https://doi.org/10.1080/10438590600984026

Belenzon, S., \& Berkovitz, T. (2007). Innovation in Business Groups. Centre for Economic Performance, LSE, CEP Discussion Papers, 56. https://doi.org/10.2139/ssrn.1026110

Benhabib, J., \& Spiegel, M. (2005). Human Capital and Technology Diffusion. In P. Aghion \& S. Durlauf (Eds.), Handbook of Economic Growth (1st ed., Vols. 1, Part A, pp. 935-966). Elsevier. https://econpapers.repec.org/RePEc:eee:grochp:1-13

Boldrin, M., \& K Levine, D. (2012). The Case Against Patents. Journal of Economic Perspectives, 27. https://doi.org/10.2139/ssrn.2148738

Bordogna, J., Fromm, E., \& Ernst, E. W. (1993). Engineering Education: Innovation Through Integration. Journal of Engineering Education, 82(1), 3-8. https://doi.org/10.1002/j.2168-9830.1993.tb00065.x

Bougrain, F., \& Haudeville, B. (2002). Innovation, collaboration and SMEs internal research capacities. Research Policy, 31(5), 735-747. https://doi.org/https://doi.org/10.1016/S00487333(01)00144-5

Bruton, G. D., \& Rubanik, Y. (2002). Resources of the firm, Russian high-technology startups, and firm growth. Journal of Business Venturing, 17(6), 553-576. https://doi.org/https://doi.org/10.1016/S08839026(01)00079-9

Buesa, M., Baumert, T., Heijs, J., \& Martinez, M. (2019). Los factores determinantes de la innovación: un análisis econométrico.

Chaminade, C., \& Vang, J. (2008). Globalisation of knowledge production and regional innovation policy: Supporting specialized hubs in the Bangalore software industry. Research Policy, 37(10), 1684-1696. https://doi.org/https://doi.org/10.1016/j.respol.2008.08. 014

Coe, D. T., Helpman, E., \& Hoffmaister, A. W. (2009). International R\&D spillovers and institutions. European Economic Review, 53(7), 723-741. https://doi.org/https://doi.org/10.1016/j.euroecorev.200 9.02.005

Cohen, W. M. (2010). Fifty Years of Empirical Studies of Innovative Activity and Performance (Vol. 1, pp. 129213). Elsevier. https://econpapers.repec.org/RePEc:eee:haechp:v1_129

Cornel SC Johnson College of Bussines. (2019). The Global Innovation Index.

D. Arthurs, J., \& Busenitz, L. (2006). Dynamic Capabilities and Venture Performance: The Effects of Venture Capitalists. Journal of Business Venturing, 21, 195215. https://doi.org/10.1016/j.jbusvent.2005.04.004

Damanpour, F., \& Gopalakrishnan, S. (2001). The Dynamics of the Adoption of Product and Process Innovations in Organizations. Journal of Management Studies, 38, 4565. https://doi.org/10.1111/1467-6486.00227

Dougherty, D., \& Heller, T. (1994). The Illegimacy of Successful Product Innovation in Established Firms. Organization Science - ORGAN SCI, 5, 200-218. https://doi.org/10.1287/orsc.5.2.200

Drucker, P. (1985). Innovation and Entrepreneurship: Practice and Principles. Public Productivity Review, 10. https://doi.org/10.2307/3380320

E. Porter, M., \& Stern, S. (2000). Measuring the "Ideas" Production Function: Evidence from International Patent Output.

Fagerberg, J. (1988). International Competitiveness. Economic Journal, 98(391), 355-374. https://econpapers.repec.org/RePEc:ecj:econjl:v:98:y:1 988:i:391:p:355-74

Fagerberg, J., \& Verspagen, B. (2002). Technology-gaps, innovation-diffusion and transformation: an evolutionary interpretation. Research Policy, 31(8), 1291-1304.

https://doi.org/https://doi.org/10.1016/S00487333(02)00064-1

Fisman, R., \& Khanna, T. (2004). Facilitating Development: The Role of Business Groups. World Development, 32(4), 609-628.

https://doi.org/https://doi.org/10.1016/j.worlddev.2003. 08.012

Freeman, C. (1988). Technology policy and economic performance; lessons from Japan: Christopher Freeman, (Frances Printer Publishers, London, New York, 1987) pp. 155, [UK pound]20.00. Research Policy, 17(5), 309-310.

Gans, J. S., \& Stern, S. (2003). The product market and the market for "ideas": commercialization strategies for technology entrepreneurs. Research Policy, 32(2), 333 350. https://doi.org/https://doi.org/10.1016/S00487333(02)00103-8

Gedik, Y. (2012). Geographical Localisation of Knowledge Spillovers by Australian Patent Citations*. Economic Papers: A Journal of Applied Economics and Policy, 31(2), 173-181. https://doi.org/10.1111/j.17593441.2012.00172.x 
Griliches, Z. (1979). Issues in Assessing the Contribution of R\&D to Productivity Growth. Bell Journal of Economics, 10, 92-116. https://doi.org/10.2307/3003321

Hagedoorn, J. (2002). Inter-Firm R\&D Partnerships: An Overview of Major Trends and Patterns Since 1960. Research Policy, 31, 477-492. https://doi.org/10.1016/S0048-7333(01)00120-2

Hall, B. H., \& Lerner, J. (2010). Chapter 14 - The Financing of R\&D and Innovation. In B. H. Hall \& N. Rosenberg (Eds.), Handbook of The Economics of Innovation, Vol. 1 (Vol. 1, pp. 609-639). North-Holland. https://doi.org/https://doi.org/10.1016/S01697218(10)01014-2

Hall, O. (1967). Medical Innovation: A Diffusion Study. By James S. Coleman, Elihu Katz, and Herbert Menzel. Foreword by Joseph A. Precker. Indianapolis: The Bobbs-Merrill Company, 1966. 246 pp. Illustrated. II\$2.95. Paper. Social Forces, 46(2), 291. https://doi.org/10.2307/2574625

Hausmann, R., \& Rodrik, D. (2003). Economic development as self-discovery. Journal of Development Economics, 72(2), 603-633.

https://doi.org/https://doi.org/10.1016/S03043878(03)00124-X

Heckman, J. (1977). Sample Selection Bias As a Specification Error (with an Application to the Estimation of Labor Supply Functions). National Bureau of Economic Research, Inc, NBER Working Papers.

Hobday, M. (2005). Firm-Level Innovation Models: Perspectives on Research in Developed and Developing Countries. Technology Analysis and Strategic Management, 17. https://doi.org/10.1080/09537320500088666

Hollander, S. (2007). The Sources of Increased Efficiency: A Study of DuPont Rayon Plants. In Technology and Culture (Vol. 7). https://doi.org/10.2307/1054889

Huergo, E., \& Moreno, L. (2015). National or international public funding? Subsidies or loans? Evaluating the innovation impact of $R \& a m p ; D$ support programmes. https://econpapers.repec.org/RePEc:pra:mprapa:64926

Iammarino, S., Sanna-Randaccio, F., \& Savona, M. (2007). The perception of obstacles to innovation. Multinational and domestic firms in Italy.

Instituto Nacional de Estadísticas y Censos/Senecyt. (2015). Encuesta de Actividades de Ciencia, Tecnología e Innovación. 64.

Jaffe, A. (1986). Technological Opportunity and Spillovers of R\&amp;D: Evidence from Firms' Patents, Profits, and Market Value. American Economic Review, 76(5), 984-1001. https://econpapers.repec.org/RePEc:aea:aecrev:v:76:y:1 986:i:5:p:984-1001

Jameson, F. (2016). Third-World Literature in the Era of Multinational Capitalism (pp. 71-90). https://doi.org/10.1002/9781119118589.ch5

Jovanovic, B., \& Rob, R. (1989). The Growth and Diffusion of Knowledge. Review of Economic Studies, 56, 569582. https://doi.org/10.2307/2297501

K. Whitaker, J., \& D. Nordhaus, W. (1970). Invention, Growth and Welfare: A Theoretical Treatment of
Technological Change. Economica, 37, 443. https://doi.org/10.2307/2551965

Kaldor, M., Sharp, M., \& Walker, W. (1986). Industrial competitiveness and Britain's defence. Lloyd's Bank Review, 162, 31-49.

Keller, W. (1997). How trade patterns and technology flows affect productivity growth, Working Paper num. 6990.

Kellman, M. (1983). Relative Prices and International Competitiveness: An Empirical Investigation. Empirical Economics, 8, 125-138. https://doi.org/10.1007/BF01969339

Kenessey, Z. (1987). The primary, secondary, tertiary and quaternary sectors of the economy. Review of Income and Wealth, 33, 359-385. https://doi.org/10.1111/j.1475-4991.1987.tb00680.x

Kim, L., of Learned Societies, A. C., Institute, C. U. E. A., \& Press, H. B. (1997). Imitation to Innovation: The Dynamics of Korea's Technological Learning. Harvard Business School Press. https://books.google.com.ec/books?id=ey2ZoWyc2jQC

Kimberly, J. R., \& Evanisko, M. J. (1981). Organizational Innovation: The Influence of Individual, Organizational, and Contextual Factors on Hospital Adoption of Technological and Administrative Innovations. Academy of Management Journal, 24(4), 689-713. https://doi.org/10.5465/256170

Klepper, S., \& M Cohen, W. (1996). A reprise of size and R\&D. Economic Journal, 106, 925-951. https://doi.org/10.2307/2235365

Lamberton, D. M. (1986). The Positive Sum Strategy: Harnessing Technology for Economic Growth edited by Ralph Landau and Nathan Rosenberg (National Academy Press, Washington, D.C., 1986) pp. xiv + 640, \$35.00, ISBN 0-309-03630-5. Prometheus, 4(1), 214-219. https://doi.org/10.1080/08109028608629601

Landau, R., \& Rosenberg, N. (Eds.). (1986). The Positive Sum Strategy: Harnessing Technology for Economic Growth. The National Academies Press. https://doi.org/10.17226/612

Lane, P., \& Lubatkin, M. (1998). Relative Absorptive Capacity and Inter-Organizational Learning. Strategic Management Journal, 19, 461-477. https://doi.org/10.1002/(SICI)10970266(199805)19:5<461::AID-SMJ953>3.0.CO;2-L

Lenox, M., \& King, A. (2004). Prospects for Developing Absorptive Capacity through Internal Information Provision. Strategic Management Journal, 25, 331345. https://doi.org/10.1002/smj.379

Lileeva, A., \& Trefler, D. (2010). Improved Access to Foreign Markets Raises Plant-level Productivity...For Some Plants*. The Quarterly Journal of Economics, 125(3), 1051-1099. https://doi.org/10.1162/qjec.2010.125.3.1051

Liu, X., Hodgkinson, I., \& Chuang, F. (2013). Foreign competition, domestic knowledge base and innovation activities: Evidence from Chinese high-tech industries. Research Policy, 43. https://doi.org/10.1016/j.respol.2013.11.005

Llanes, G., \& Stefano, T. (2010). Patent policy, patent pools, and the accumulation of claims in sequential innovation. Econ Theory, 703-725.

Lucas, R. (1989). On The Mechanics Of Economic 
Development. Journal of Monetary Economics, 22, 342. https://doi.org/10.1016/0304-3932(88)90168-7

Lugones, G., Suárez, D., \& Gregorini, S. (2019). DOCUMENTOS DE TRABAJO LA INNOVACIÓN COMO FÓRMULA PARA MEJORAS COMPETITIVAS COMPATIBLES CON INCREMENTOS SALARIALES. EVIDENCIAS EN EL CASO ARGENTINO.

Lundvall, B.-Å. (2019). Higher Education, Innovation and Economic Development.

M. Cohen, W., \& Levinthal, D. (1990). Absorptive Capacity: A New Perspective on Learning and Innovation. Administrative Science Quarterly, 35, 128-152. https://doi.org/10.2307/2393553

Mansfield, E. (1986). Patents and Innovation: An Empirical Study. Management Science, 32, 173-181. https://doi.org/10.1287/mnsc.32.2.173

Mansfield, E., Schwartz, M., \& Wagner, S. (1981). Imitation Costs and Patents: An Empirical Study. Economic Journal, 91, 907-918. https://doi.org/10.2307/2232499

Maskus, K. (2000). Intellectual property rights and economic development. Case Western Reserve Journal of International Law, 32, 471-506.

Merges, R., \& R. Nelson, R. (1990). The Complex Economics of Patent Scope. Columbia Law Review, 90. https://doi.org/10.2307/1122920

Metcalfe, S., \& Miles, I. (2000). Innovation Systems in the Service Economy: Measurement and Case Study Analysis (Vol. 18). https://doi.org/10.1007/978-1-46154425-8

Myers, S. C., \& Majluf, N. S. (1984). Corporate financing and investment decisions when firms have information that investors do not have. Journal of Financial Economics, 13(2), 187-221. https://doi.org/https://doi.org/10.1016/0304405X(84)90023-0

Nadiri, M. (1993). Innovations and Technological Spillovers. 4423.

Naranjo-Valencia, J. C., Jiménez, D. J., \& Sanz-Valle, R. (2012). ¿Es la cultura organizativa un determinante de la innovación en la empresa? Cuadernos de Economía y Dirección de La Empresa, 15(2), 63-72. https://doi.org/https://doi.org/10.1016/j.cede.2011.07.0 04

Narula, R., \& Zanfei, A. (2003). Globalisation of Innovation The Role of Multinational Enterprises. In DRUID, Copenhagen Business School, Department of Industrial Economics and Strategy/Aalborg University, Department of Business Studies, DRUID Working Papers.

Nelson, R. (1959). The Simple Economics of Basic Scientific Research. Journal of Political Economy, 67(3), 297306. https://doi.org/10.1086/258177

Nelson, R., \& Phelps, E. (1965). Investment in Humans, Technological Diffusion and Economic Growth (Issue 189). https://econpapers.repec.org/RePEc:cwl:cwldpp:189

Nobel, R., \& Birkinshaw, J. (1998). Innovation in Multinational Corporations: Control and Communication Patterns in International R\&D Operations. Strategic Management Journal, 19. https://doi.org/10.1002/(SICI)10970266(199805)19:5<479::AID-SMJ954>3.0.CO;2-U
Petersen, B., \& Himmelberg, C. (1994). R \& D and Internal Finance: A Panel Study of Small Firms in High-Tech Industries. The Review of Economics and Statistics, 76, 38-51. https://doi.org/10.2307/2109824

Piga, C., \& Vivarelli, M. (2004). Internal and External R\&D: A Sample Selection Approach*. Oxford Bulletin of Economics and Statistics, 66, 457-482. https://doi.org/10.1111/j.1468-0084.2004.00089.x

Porter, M. (1990). The Competitive Advantage Of Nations. In Harvard Business Review (Vol. 68). https://doi.org/10.1007/978-1-349-11336-1

Rai, R. K., \& Jagannathan, S. (2012). Do Business Method Patents Encourage Innovation? (Boston College Intellectual Property \& Technology Forum (Ed.)).

Raspe, O. (2009). The Regional Knowledge Economy; a Multilevel Perspective on Firm Performance and Localized Knowledge Externalities.

Rogers, E. (1962). Diffusion of Innovations (pp. 418-434). https://doi.org/10.4324/9780203710753-35

Rogers, M. (2004). Networks, Firm Size and Innovation. Small Business Economics, 22, 141-153. https://doi.org/10.1023/B:SBEJ.0000014451.99047.69

Rosenberg, N. (1972). Factors affecting the diffusion of technology. Explorations in Economic History, 10, 3 33. https://doi.org/10.1016/0014-4983(72)90001-0

Rosenberg, N. (1984). Inside the Black Box: Technology and Economics. Technology and Culture, 25. https://doi.org/10.2307/2596875

Savic, M., Lawton-Smith, H., \& Bournakis, I. (2014). The effect of external knowledge sources and their geography on innovation in Knowledge Intensive Business Services (KIBS) SMEs; some implications for de-industrialised regions in the $U K$.

Schumpeter, J. (1934). The Theory of Economic Development: An Inquiry Into Profits, Capital, Credit, Interest, and the Business Cycle. 55.

Seru, A. (2007). Do Conglomerates Stifle Innovation? SSRN Electronic Journal. https://doi.org/10.2139/ssrn.1674018

Spielkamp, A., \& Rammer, C. (2009). Financing of innovations - thresholds and options. Management \& Marketing, 4.

Symeonidis, G. (1996). Innovation, Firm Size and Market Structure. 161.

https://doi.org/https://doi.org/https://doi.org/10.1787/60 3802238336

Tether, B. (1998). Small and Large Firms: Sources of Unequal Innovations? Research Policy, 27, 725-745. https://doi.org/10.1016/S0048-7333(98)00079-1

Tether, B. S., \& Tajar, A. (2008). Beyond industry-university links: Sourcing knowledge for innovation from consultants, private research organisations and the public science-base. Research Policy, 37(6), 10791095.

https://doi.org/https://doi.org/10.1016/j.respol.2008.04. 003

Veugelers, R. (1996). Internal R\&D Expenditures and External Technology Sourcing. Research Policy, 26, 303-315. https://doi.org/10.1016/S00487333(97)00019-X 
Yeaple, S., Helpman, E., \& J. Melitz, M. (2004). Export Versus FDI with Heterogeneous Firms. American Economic Review, 94, 300-316.

https://doi.org/10.1257/000282804322970814

\section{BIOGRAFÍAS}

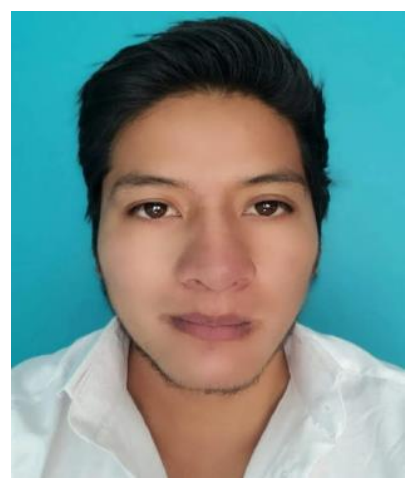

Danny Eduardo Pilamunga

Shaca, graduado como Ingeniero en Ciencias Económicas y Financieras de la Escuela Politécnica Nacional, nacido en la comunidad de Echaleche-Pilahuin el 03 de Enero de 1995. Domina cuatro idiomas, entre ellos el chinomandarin y el kichwa. Actualmente trabaja en proyectos empresariales independientes y se desempeña como analista de riesgos económicos y financieros en la Cooperativa de Ahorro y Crédito Mushuc Runa. ORCID: 0000-0001-5833-7026

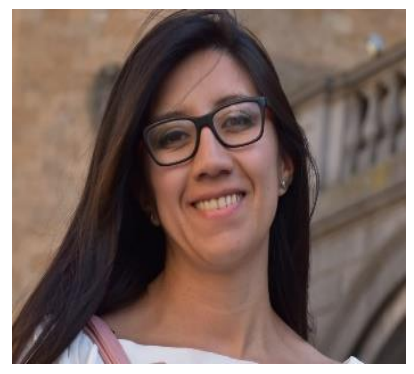

Grace Carolina GuevaraRosero, es Doctora en Economía por la Universidad Jean Monnet de Saint-Etienne de Francia. Obtuvo su Máster en Game Theory, Experimental Economics and Applied Econometrics en el 2012. Actualmente es docente del Departamento de Economía Cuantitativa de la Escuela Politécnica Nacional. Ha colaborado en insitituciones como la Secretaría Nacional de Planificación y Desarrollo, la Secretaría Nacional de Educación Superior, Ciencia y Tecnología, entre otras. Sus áreas de investigación son la Economía Regional y Urbana, la Economía de la Innovación y Orgnaización Industrial. ORCID: 0000-0001-7605-1443 